\title{
Results of the Ha Survey of the Small Magellanic Cloud
}

\author{
Margarita Rosado \\ Instituto de Astronomía, UNAM. Ap. Postal 70-264, CP04510 México, \\ D.F.
}

\author{
Etienne le Coarer \\ Observatoire de Grenoble. BP $\{3 X$ Grenoble CEDEX, France.
}

Yvon Georgelin

Observatoire de Marseille. 2 Place Le Verrier, 13248 Marseille Cedex \&, France.

\begin{abstract}
We present the results of a kinematic survey of the Small Magellanic Cloud, performed with a scanning FP interferometer mainly at $\mathrm{H} \alpha$. These data allow us to study the overall dynamics of the ionized hydrogen of this galaxy as well as the kinematics of classes of objects. In this presentation we exemplify how the availability of data in several wavelengths can be very useful in establishing the nature of large nebular complexes of the Small Magellanic Cloud. Our kinematic study allows us to discriminate faint shocked nebulae (such as supernova remnants) inside bright $\mathrm{HII}$ regions. This is confirmed by recently available radio and X-ray data.
\end{abstract}

\section{Introduction}

The Small Magellanic Cloud (SMC) is one of the nearest galaxies to the Milky Way. Its controversial global dynamics and the possibility of studying in a global context the interactions of young stars and interstellar matter have motivated us to undertake a study of the kinematics of the ionized gas in the SMC. In order to do this, we have completed a kinematic survey of the entire galaxy by means of the H $\alpha$ Survey equipment of the Marseille Observatory.

\section{Main Characteristics of the $\mathbf{H} \alpha$ Survey and Observations}

The $\mathrm{H} \alpha$ Survey equipment consists of a $36 \mathrm{~cm}$ diameter telescope equipped with a focal reducer, two scanning FP interferometers, a series of interference filters and a photon counting camera. Table 1 in Le Coarer et al. (1992), gives the general characteristics of this equipment. We obtain FP data cubes similar to radio data cubes $(x, y, \lambda)$ which have an angular resolution of $9^{\prime \prime}$ and a field of view of $38^{\prime}$ (covered by the $256 \times 256$ pixels of the photon counting camera). The sampling spectral resolutions are of 5 and $16 \mathrm{~km} \mathrm{~s}^{-1}$ at $\mathrm{H} \alpha$, and the free 
spectral ranges are 115 and $376 \mathrm{~km} \mathrm{~s}^{-1}$ at $\mathrm{H} \alpha$ respectively, depending on the scanning FP interferometer used. The limiting emission measure for detection is estimated to be

$$
E M_{\mathrm{lim}}=1 \mathrm{~cm}^{-6} \mathrm{pc}
$$

(for a $T=10^{4} \mathrm{~K}$, Case B photoionized nebula), reached in an exposure time of $8280 \mathrm{~s}$. In the observations of the SMC we have covered the whole galaxy by overlapping the $\mathbf{3 8}^{\prime}$ field frames in such a way that some HII regions already observed could be used as photometric calibrators of the $\mathrm{H} \alpha$ fluxes.

\section{Main Results}

These observations have allowed us to obtain the following results:

1. An HII region catalogue of 143 of the 167 DEM HII regions of the SMC ( Le Coarer et al., 1993). This catalogue includes radial velocities, velocity dispersions and $\mathrm{H} \alpha$ fluxes of each HII region. In addition to this, we have, still unpublished, an isophote map and the whole velocity field for all the HII regions of the catalogue.

2. The detection and kinematic knowledge of faint ionized hydrogen in the SMC. Indeed, our observations reveal that the HII regions are embedded in a bath of ionized hydrogen which correlates well with the IR emission detected by IRAS at $60 \mu$. Thus, this faint ionized $H$ traces the dust distribution. The kinematics of this ionized $H$ is similar to the one found for the neutral $H$.

3. The velocity field of the ionized $H$ in the entire SMC. This reveals the complexities in the kinematics of this galaxy; nevertheless some indication of rotation is found along the bar.

4. The development of a kinematic method in order to detect shocked nebulae (as supernova remnants (SNRs), and wind-blown bubbles). Those nebulae, appearing in several of our $\lambda$-maps, are formed by shocks of the interstellar medium (Rosado et al., 1994).

\section{Detection of Several SNRs Inside Large Nebular Complexes}

\subsection{The SNRs Inside the HII Region N19 in the SMC}

The nebular complex N19 has linear dimensions of about $110 \mathrm{pc}$ (assuming a distance to the SMC of $65 \mathrm{kpc}$ ) and is presumably formed by a rich OB stellar association. Previous studies have shown that one SNR is located inside this nebula towards the western boundary. However, our kinematic data at $\mathrm{H} \alpha$ and [SII] reveal that there are two other SNRs inside the HII region and two other SNR candidates. We have obtained velocity profiles integrated over windows of $45^{\prime \prime}$ side. We have identified at least three zones well delimited, where the velocity profiles are complex indicating shock velocities up to $170 \mathrm{~km} \mathrm{~s}^{-1}$. These zones, of at most $40 \mathrm{pc}$ size, are separated entities one of which coincides in morphology with the previously reported SNR 0045-73.4. In addition, these zones have high [SII]/Ha line-ratios. The splitting pattern in each zone confirms the idea of three separate entities (i.e., three SN explosions) instead of one SN explosion in an already formed three-lobed cavity (Rosado et al., 1994). On the other hand, the energy required to form a larger structure (radius of $60 \mathrm{pc}$ ), 


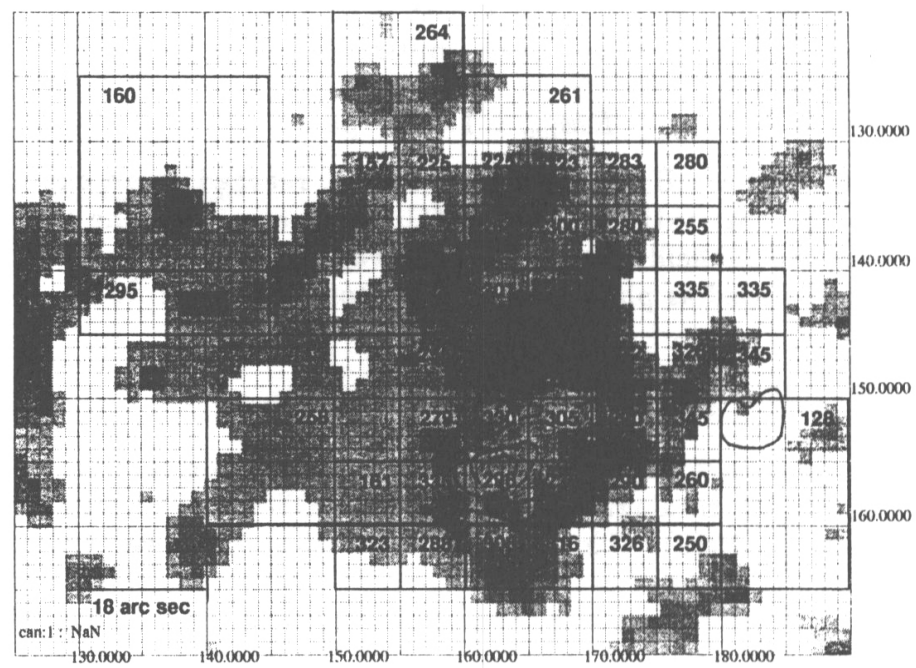

Figure 1. [SII $] / \mathrm{H} \alpha$ line-ratio image of N19. Superimposed on it we have marked the differences in velocities (in $\mathrm{km} \mathrm{s}^{-1}$ ) obtained from our data and ROSAT isophotes.

expanding at $170 \mathrm{~km} \mathrm{~s}^{-1}$, in a medium of ambient density $1 \mathrm{~cm}^{-3}$, is an order of magnitude higher than the energy supplied to the ISM by a single SN explosion. Thus we have several pieces of evidence supporting the multi-SN hypothesis.

These kinematic identifications were confirmed latter when observations in other wavelengths were available. Figures 1 and 2 show our identified zones and superimposed on them the X-ray (from ROSAT results) and the non-thermal radio emission (obtained from the "scaled subtraction method", Ye, 1987) maps. These maps were kindly supplied by Dr. Y.H. Chu (Chu, 1994). As we can see from these figures, our optical kinematic identifications have counterparts in the X-ray or radio domains or both. In addition, the morphology of the separated zones, revealed by our method agrees with their morphology in other wavelengths. It is interesting to note, for example, that in Fig. 1, the X-ray emission of the SNR 0045-73.4 is confined to the interior of the optical filaments with enhanced $[\mathrm{SII}] / \mathrm{H} \alpha$ line-ratios reported by us.

\subsection{The SNRs Associated With the HII Region N66 in the SMC}

N66 is the largest and brightest HII region in the SMC. Its diameter is of about $250 \mathrm{pc}$ and this nebula is excited by a rich cluster of very early-type stars.

Previous studies report one SNR identified at the south-west boundary (SNR 0056-72.5) and an additional SNR located near the center, identified both by the "scaled subtraction method" and by its kinematics (Ye et al. 1991). The latter studies suggest another SNR candidate located at the western boundary.

The velocity profiles of the interesting zones mentioned before show that violent motions are present in the three nebulosities, revealed by the existence of high velocity wings superposed to a quite high HII region emission profile. 


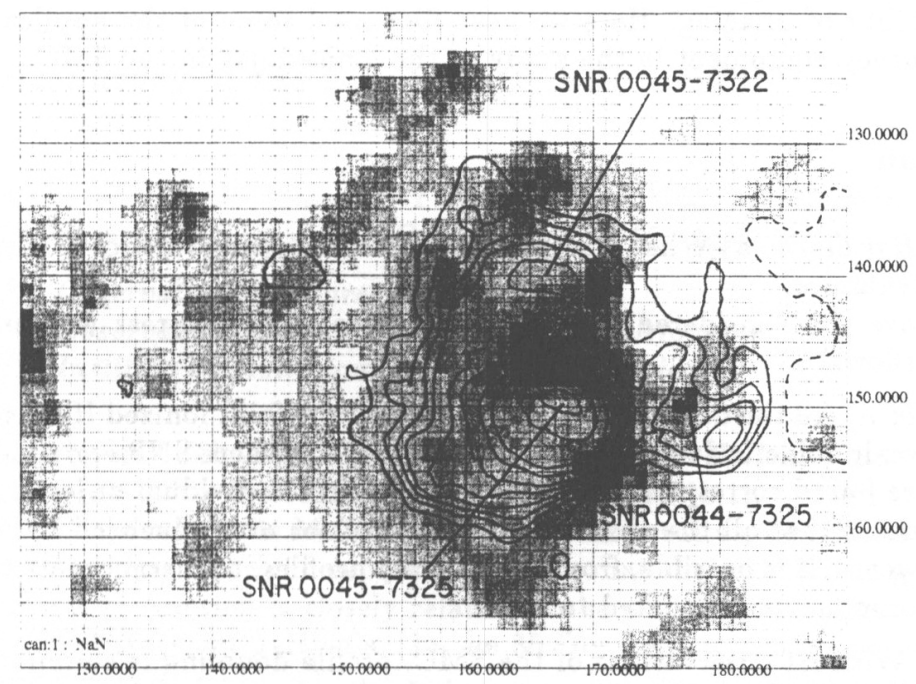

Figure 2. [SII] $/ \mathrm{H} \alpha$ line-ratio image of N19. Superimposed on it are the non-thermal radio emission (Ye 1987).

\subsection{Discussion}

We have presented two examples where large HII complexes have several SNRs and wind-blown bubbles inside them.

In those cases, SNR identification cannot be accomplished by classical methods. Instead, it is seen that the "kinematic method" (Rosado el al. 1994) and the "scaled subtraction method" (Ye et al. 1991) are useful tools in discriminating the SNR emission against stronger HII region emission.

These nebular complexes are excellent laboratories where SNR interactions can be studied. These are examples of medium size HII complexes where multiSN events have been found. Indeed, the fact of finding several SNRs inside these HII complexes is an important clue in the understanding of the formation of superbubbles (typical diameters of $1 \mathrm{kpc}$ ) in external galaxies. Several theories have been developed for explaining their formation and energetics: combined action of winds and multi-SN, cloud collisions with the galactic disk, sequential star formation, etc.. The study of the realistic conditions of multi-SN events forming medium size complexes could help in the discrimination between the models mentioned above.

On the other hand, in the two cases discussed, we have triplicated the number of SNRs detected. This implies that after a careful kinematic survey, complemented by radio and X-ray observations, the SN rates of irregular galaxies (and perhaps of spirals too) should be revised.

\section{Conclusions}

Scanning FP interferometers are very useful in the study of the kinematics of nearby galaxies due to their high spectral resolution, their high field of view and 
the possibility of imaging. Here we have discussed some of the applications of the $\mathrm{H} \alpha$ Survey equipment in the study of the ionized gas in the SMC.

\section{Discussion}

J. Bland-Hawthorn: a) What fraction of your data shows evidence of large-scale galactic dynamics?

b) Have you considered which emission line might supress the energetics due to outflows?

M. Rosado: a) All the data on HII regions and on the faint ionized $\mathrm{H}$ must reflect the large-scale galactic dynamics. Only those regions where SNRs or wind-blown bubbles are found correspond to internal motions of individual objects

b) I think that all the observable emission lines are influenced by the outflows. However, it is possible, from the velocity profiles, to discriminate the high velocity components associated to them.

B. Tully: With HI observations of the SMC, profile doubling is common and it has been proposed that there are multiple components in these lines of sight. Do you also see profile splitting and, if so, is there agreement with the HI information?

M. Rosado: We have seen (Le Coarer et al., 1993) that the faint ionized $H$ has a multi- velocity structure similar the one found for the HI. HII regions, on the other hand, have a single velocity component which, in general, coincides with the velocity of the main HI peak located at that position.

H. Castañeda: There are notorious problems in the detection of SNRs within HII regions. Is the kinematic data very efficient finding evidence for SNR, or do you feel that ROSAT data is more appropiated?

M. Rosado: The observations in several wavelengths are complementary. We see from Fig. 1 and 2 in this presentation that one of the SNR in N19 is missing in X-rays whereas it appears in radio and with our kinematic method. Other examples could be given in the other sense I suppose. This method is quite useful for external galaxies.

\section{References}

Chu, Y.H. 1994, private communication

Le Coarer, E., Amram, P., Boulesteix, J. et al. 1992, A\&A, 257, 389

Le Coarer, E., Rosado, M., Georgelin, Y.P. et al. 1993, A\&A, 280, 365

Rosado, M., Le Coarer, E., Georgelin, Y.P. 1994, A\&A, in press

Ye, T. 1987, Ph.D. Thesis.

Ye, T., Turtle, A.J., Kennicutt, R.C. 1991, MNRAS, 249, 722 